\title{
CONDITIONAL DISTRIBUTIONS OF PROCESSES RELATED TO FRACTIONAL BROWNIAN MOTION
}

\author{
HOLGER FINK* AND \\ CLAUDIA KLÜPPELBERG, ${ }^{* *}$ Technische Universität München \\ MARTINA ZÄHLE, ${ }^{* * *}$ University of Jena
}

\begin{abstract}
Conditional distributions for affine Markov processes are at the core of present (defaultable) bond pricing. There is, however, evidence that Markov processes may not be realistic models for short rates. Fractional Brownian motion (FBM) can be introduced by an integral representation with respect to standard Brownian motion. Using a simple prediction formula for the conditional expectation of an FBM and its Gaussianity, we derive the conditional distributions of FBM and related processes. We derive conditional distributions for fractional analogies of prominent affine processes, including important examples like fractional Ornstein-Uhlenbeck or fractional CoxIngersoll-Ross processes. As an application, we propose a fractional Vasicek bond market model and compare prices of zero-coupon bonds to those achieved in the classical Vasicek model.

Keywords: Affine process; conditional characteristic function; fractional affine process; short rate; macroeconomic variables process; fractional Brownian motion; long-range dependence; interest rate; zero-coupon bond; fractional Vasicek model; prediction

2010 Mathematics Subject Classification: Primary 60G15; 60G22; 60H10; 60H20;

$91 \mathrm{G} 30$

Secondary 60G10; $91 \mathrm{G} 60$
\end{abstract}

\section{Introduction}

Prediction problems arise in many financial and technical applications. For example, in a bond market driven by an adapted short rate process $r=(r(v))_{v \in[0, T]}$ the price of a nondefaultable zero-coupon bond with maturity $t \geq 0$ at time $0 \leq s \leq t$ is given by the conditional expectation

$$
B(s, t)=\mathbb{E}^{\mathbb{Q}}\left[\exp \left\{-\int_{s}^{t} r(v) \mathrm{d} v\right\} \mid r(v), v \in[0, s]\right]
$$

under some risk-neutral measure $\mathbb{Q}$.

For a wide class of stochastic processes, in particular affine models (see, e.g. [6] and [7]), such predictions are easy to calculate and depend only on the level of the process at time $t$ due to their Markov property. However, staying in the bond framework above, Markov models may not be sufficient to catch the real market structure, as was shown in the ongoing financial crisis. One reason behind this is that short rates, which are driven by macroeconomic

\footnotetext{
Received 11 May 2010; revision received 31 July 2012.

* Postal address: Center for Mathematical Sciences, Technische Universität München, 85748 Garching, Germany. Email address: fink@ma.tum.de

** Postal address: Center for Mathematical Sciences, and Institute for Advanced Study, Technische Universität München, 85748 Garching, Germany. Email address: cklu@ma.tum.de

*** Postal address: Mathematical Institute, University of Jena, 07740 Jena, Germany.

Email address: zaehle@minet.uni-jena.de
} 
variables such as domestic gross products, supply and demand rates, and volatilities, exhibit long-range dependence, which cannot be captured by Markov models. Empirical evidence has been reported over the years and we refer the reader to [15] for details and further references. In particular, Backus and Zin [1] provided in their Section 4 evidence for long memory in the short rate process.

When it comes to modeling such structures, fractional processes, such as fractional Brownian motion (FBM), have been at the core of most models. Their non-Markovianity, however, makes prediction more complicated, since all past information will play a role. Since FBM can be introduced by an integral representation of a standard Brownian motion (which is itself an affine process), certain structures remain when deriving the conditional distribution.

In this paper we calculate the conditional distributions of FBM-driven integrals and, in general, of solutions to FBM-driven stochastic differential equations (SDEs), which have been considered by Buchmann and Klüppelberg [4] based on previous work by Zähle [22]. Our results include important models, such as fractional Ornstein-Uhlenbeck (FOU), fractional Vasicek, and fractional Cox-Ingersoll-Ross (FCIR) processes. An application is given by a bond market model, where the short rate is described by a fractional Vasicek SDE. Justified by Section 3 of Biagini et al. [3], which was based on the previous work of Guasoni et al. [12], [13] and Ohashi [17], we can construct such a model by specifying the dynamics directly under a risk-neutral measure. In particular, Biagini et al. [3] derived the fractional Vasicek model from a fractional Heath-Jarrow-Morton (HJM) approach. Ohashi [17] introduced such an HJM model and proved that it is arbitrage free under suitable transaction costs. Biagini et al. [3] extended these results to propose a fractional Vasicek model with fractional hazard rate, and explicitly calculated bond option prices. Using these results, we can directly model under a risk-neutral measure in this paper.

Our work is based on a formula for the conditional expectation of FBM, which has been derived by Gripenberg and Norros [11] and Pipiras and Taqqu [19].

Our paper is organized as follows. In Section 2 we briefly recall integration with respect to FBM and state a prediction formula for its conditional expectation. In Section 3 we present our main results on conditional distributions of FBM related processes, including important models, such as the FOU and FCIR models. An application to a bond market described by a fractional Vasicek model is presented in Section 4. Zero-coupon bond prices are calculated and compared to the classical Brownian situation. We conclude the paper with the proofs of our results in Section 5.

We will always assume a given complete probability space $(\Omega, \mathcal{F}, \mathcal{Q})$, and denote by $L^{2}(\Omega)$ the space of square-integrable random variables. For a family of random variables $(X(i))_{i \in I}$, $I$ some index set, let $\sigma\{X(i), i \in I\}$ denote the completion of the generated $\sigma$-algebra. The spaces of integrable and square-integrable real functions are denoted by $L^{1}(\mathbb{R})$ and $L^{2}(\mathbb{R})$, respectively. On a compact interval $[0, T]$, the corresponding function spaces are denoted by $L^{1}([0, T])$ and $L^{2}([0, T])$. Furthermore, $\|\cdot\|_{2}$ is the $L^{2}$-norm, and $\mathbb{R}_{+}$and $\mathbb{R}_{-}$are the positive and negative real half-lines, respectively.

Recall that, for $\mu \in \mathbb{R}$ and $\sigma>0$, a random variable $X$ is normally distributed with expectation $\mu$ and variance $\sigma^{2}$ if and only if $\mathbb{E}\left[\mathrm{e}^{\mathrm{i} u X}\right]=\exp \left\{\mathrm{i} u \mu-u^{2} \sigma^{2} / 2\right\}$ for $u \in \mathbb{R}$. We will repeatedly use this relationship without further comment.

\section{Preliminaries}

We recall that FBM is a zero-mean Gaussian process starting at 0 with stationary increments satisfying $\left(B^{H}(c t)\right)_{t \geq 0} \stackrel{\text { FDD }}{=} c^{H}\left(B^{H}(t)\right)_{t \geq 0}$ for every $c>0$. The parameter $H \in(0,1)$ and 
$\stackrel{\text { FDD, }}{=}$ denotes equality in finite-dimensional distributions. We also assume that $B^{H}$ is standard, i.e. that $\mathbb{E}\left[B^{H}(1)^{2}\right]=1$. For general background on FBM, we refer the reader to [20]. For the present paper, we will draw heavily from Pipiras and Taqqu [18], [19].

It is appropriate in our context to use fractional calculus, which suggests replacing $H$ by the fractional parameter $\kappa=H-\frac{1}{2} \in\left(-\frac{1}{2}, \frac{1}{2}\right)$. The case $\kappa \in\left(0, \frac{1}{2}\right)$ refers to long-range dependence. We also recall that $\kappa=0$ refers to standard Brownian motion and we will write $B^{0}=B$.

Throughout the paper, we will work on the compact interval $[0, T]$ for some $T>0$ and define the fractional Riemann-Liouville integral with finite time horizon for $\kappa>0$ :

$$
\left(I_{T-}^{\kappa} f\right)(s)=\frac{1}{\Gamma(\kappa)} \int_{s}^{T} f(r)(r-s)^{\kappa-1} \mathrm{~d} r, \quad 0 \leq s \leq T .
$$

For $f \in L^{1}(\mathbb{R})$, this always exists almost everywhere; cf. Equation (7) of [22]. We will also need the fractional derivative with finite time horizon for $\kappa \in(0,1)$ :

$$
\left(D_{T-}^{\kappa} g\right)(u)=\frac{1}{\Gamma(1-\kappa)}\left(\frac{g(u)}{(T-u)^{\kappa}}+\kappa \int_{u}^{T} \frac{g(u)-g(s)}{(s-u)^{\kappa+1}} \mathrm{~d} s\right), \quad 0<u<T .
$$

As usual, we will often write $I_{T-}^{-\kappa}=D_{T-}^{\kappa}$. For $\kappa=0$, we set $I_{T-}^{\kappa}=D_{T-}^{\kappa}=\mathrm{id}$.

Possible spaces of integrands for FBM have been introduced in [18] and [19]:

$$
\widetilde{\Lambda}_{T}^{\kappa}:=\left\{\begin{aligned}
&\{f:[0, T] \rightarrow \mathbb{R} \mid\left.\int_{0}^{T}\left[s^{-\kappa} I_{T-}^{\kappa}\left((\cdot)^{\kappa} f(\cdot)\right)(s)\right]^{2} \mathrm{~d} s<\infty\right\}, \quad \kappa \in\left(0, \frac{1}{2}\right), \\
&\{f:[0, T] \rightarrow \mathbb{R} \mid \text { there exists } \phi_{f} \in L^{2}[0, T] \text { such } \\
&\text { that } \left.f(s)=s^{-\kappa} I_{T-}^{-\kappa}\left((\cdot)^{\kappa} \phi_{f}(\cdot)\right)(s)\right\}, \quad \kappa \in\left(-\frac{1}{2}, 0\right) .
\end{aligned}\right.
$$

In light of Lemma 4.3 of [2] we will adjust these spaces such that they are closed with respect to multiplication with an indicator function. Therefore, we define, for $\kappa \in\left(-\frac{1}{2}, \frac{1}{2}\right)$,

$$
\Lambda_{T}^{\kappa}:=\left\{f:[0, T] \rightarrow \mathbb{R} \mid \text { for all }[s, t] \subseteq[0, T]: f \mathbf{1}_{[s, t]} \in \tilde{\Lambda}_{T}^{\kappa}\right\}
$$

For $\kappa=0$, both spaces coincide and are equal to $L^{2}([0, T])$. For $\kappa \in\left(-\frac{1}{2}, \frac{1}{2}\right)$ and $f, g \in \Lambda_{T}^{\kappa}$, define the scalar product

$$
\langle f, g\rangle_{\kappa, T}:=\frac{\pi \kappa(2 \kappa+1)}{\Gamma(1-2 \kappa) \sin (\pi \kappa)} \int_{0}^{T} s^{-2 \kappa}\left[I_{T-}^{\kappa}\left((\cdot)^{\kappa} f(\cdot)\right)(s)\right]\left[I_{T-}^{\kappa}\left((\cdot)^{\kappa} g(\cdot)\right)(s)\right] \mathrm{d} s,
$$

where we set $\langle f, g\rangle_{\kappa, T}=\langle f, g\rangle_{L^{2}}$ for $\kappa=0$. Denote the corresponding norm by $\|\cdot\|_{\kappa, T}$. For $\kappa=0$, we have $\|\cdot\|_{0, T}=\|\cdot\|_{2}$. If $c$ is a step function, $\int_{0}^{T} c(s) \mathrm{d} B^{\kappa}(s)$ can be reduced to a finite sum. We then have the isometry

$$
\left\|\int_{0}^{T} c(s) \mathrm{d} B^{\kappa}(s)\right\|_{2}=\|c(\cdot)\|_{\kappa, T}
$$

and, by using approximating sequences of step functions, integration for general $c \in \Lambda_{T}^{\kappa}$ is defined in the $L^{2}$-sense, while (2.1) still holds true; cf. [19, Theorems 4.1 and 4.2].

Let $\overline{\operatorname{sp}}_{[0, T]}\left(B^{\kappa}\right)$ be the closure in $L^{2}(\Omega)$ of all possible linear combinations of the increments of FBM on $[0, T]$. Assume that we want to calculate an expression for the prediction

$$
X_{t}(s, \kappa):=\mathbb{E}\left[B^{\kappa}(t) \mid B^{\kappa}(v), v \in[0, s]\right], \quad 0 \leq s \leq t .
$$


If $X_{t}(s, \kappa) \in \overline{\operatorname{sp}}_{[0, s]}\left(B^{\kappa}\right)$, we would hope that there exists some function $c \in \Lambda_{T}^{\kappa}$ such that $X_{t}(s, \kappa)=\int_{0}^{s} c(v) \mathrm{d} B^{\kappa}(v)$. This is not immediately clear because it has been shown in [19] that, while, for $\kappa \in\left(-\frac{1}{2}, 0\right]$, the space $\left(\Lambda_{T}^{\kappa},\langle,\rangle_{\kappa, T}\right)$ is complete, i.e. a Hilbert space, for $\kappa \in\left(0, \frac{1}{2}\right)$, this is not true. However, it has been shown by Gripenberg and Norros [11, Theorem 3.1] that such a suitable $c$ still exists for $\kappa \in\left(0, \frac{1}{2}\right)$. An explicit formula for $c$ has been calculated. In fact, Theorem 7.1 of [19] shows that the same formula holds for $\kappa \in\left(-\frac{1}{2}, 0\right]$.

Lemma 2.1. Let $0 \leq s \leq t \leq T$ and $\kappa \in\left(-\frac{1}{2}, \frac{1}{2}\right)$. Then

$$
\mathbb{E}\left[B^{\kappa}(t) \mid B^{\kappa}(v), v \in[0, s]\right]=B^{\kappa}(s)+\int_{0}^{s} \Psi^{\kappa}(s, t, v) \mathrm{d} B^{\kappa}(v),
$$

where, for $v \in(0, t)$,

$$
\Psi^{\kappa}(s, t, v)=v^{-\kappa}\left(I_{s-}^{-\kappa}\left(I_{t-}^{\kappa}(\cdot)^{\kappa} \mathbf{1}_{[s, t]}(\cdot)\right)\right)(v)=\frac{\sin (\pi \kappa)}{\pi} v^{-\kappa}(s-v)^{-\kappa} \int_{s}^{t} \frac{z^{\kappa}(z-s)^{\kappa}}{z-v} \mathrm{~d} z
$$

and, for $v \in\{0, s\}$, we have $\Psi^{\kappa}(s, t, v)=0$.

If we write now

$$
\mathbb{E}\left[B^{\kappa}(t)-B^{\kappa}(s) \mid B^{\kappa}(v), v \in[0, s]\right]=\int_{0}^{s} \Psi^{\kappa}(s, t, v) \mathrm{d} B^{\kappa}(v),
$$

it is immediately clear that this prediction formula can be extended to integrals of FBM, which has been done in Lemma 1 of [8].

Proposition 2.1. For $0 \leq s \leq t \leq T$ and $\kappa \in\left(-\frac{1}{2}, \frac{1}{2}\right)$, let $c \in \Lambda_{T}^{\kappa}$. Then

$$
\mathbb{E}\left[\int_{0}^{t} c(v) \mathrm{d} B^{\kappa}(v) \mid B^{\kappa}(v), v \in[0, s]\right]=\int_{0}^{s} c(v) \mathrm{d} B^{\kappa}(v)+\int_{0}^{s} \Psi_{c}^{\kappa}(s, t, v) \mathrm{d} B^{\kappa}(v),
$$

where, for $v \in(0, s)$,

$$
\begin{aligned}
\Psi_{c}^{\kappa}(s, t, v) & =v^{-\kappa}\left(I_{s-}^{-\kappa}\left(I_{t-}^{\kappa} z^{\kappa} c(z) I_{[s, t]}(z)\right)\right)(v) \\
& =\frac{\sin (\pi \kappa)}{\pi} v^{-\kappa}(s-v)^{-\kappa} \int_{s}^{t} \frac{z^{\kappa}(z-s)^{\kappa}}{z-v} c(z) \mathrm{d} z
\end{aligned}
$$

and, for $v \in\{0, s\}$, we have $\Psi_{c}^{\kappa}(s, t, v)=0$.

In fact, all our results in this paper are a consequence of Lemma 2.1, as will be seen later.

\section{Main results}

Calculating conditional distributions by conditional characteristic functions essentially means predicting exponentials. A possible way to approach this problem for FBM-driven integrals has been considered by Duncan [8], who transformed the exponential function to a Wick exponential. While this idea works well, Proposition 2 of that paper is not correct. This can be seen immediately because its result suggests that the prediction is deterministic. The correct version with proof can be found in [9]. This has also been confirmed by our chosen approach in the present paper, which is based on the simple prediction formula of Lemma 2.1 and classical results on conditional Gaussian distributions. We want to emphasize that our approach also covers the range $\kappa \in\left(-\frac{1}{2}, 0\right)$. All proofs are postponed to Section 5 . 
For notational convenience, we fix for the rest of this section an FBM $\left(B_{t}^{\kappa}\right)_{t \in[0, T]}$ with $\kappa \in\left(-\frac{1}{2}, \frac{1}{2}\right)$. Furthermore, define

$$
\mathcal{F}_{s}:=\sigma \overline{\left\{B^{\kappa}(v), v \in[0, s]\right\}} \text { for } 0 \leq s \leq T .
$$

Theorem 3.1. Let $c \in \Lambda_{T}^{\kappa}$ and $0 \leq s \leq t \leq T$. Then $\int_{0}^{t} c(u) \mathrm{d} B^{\kappa}(u) \mid \mathscr{F}_{s}$ is normally distributed with

$$
\mathbb{E}\left[\int_{0}^{t} c(v) \mathrm{d} B^{\kappa}(v) \mid \mathcal{F}_{s}\right]=\int_{0}^{s} c(v) \mathrm{d} B^{\kappa}(v)+\int_{0}^{s} \Psi_{c}^{\kappa}(s, t, v) \mathrm{d} B^{\kappa}(v)
$$

and

$$
\operatorname{var}\left[\int_{0}^{t} c(v) \mathrm{d} B^{\kappa}(v) \mid \mathscr{F}_{s}\right]=\left\|c(\cdot) \mathbf{1}_{[s, t]}(\cdot)\right\|_{\kappa, T}^{2}-\left\|\Psi_{c}^{\kappa}(s, t, \cdot) \mathbf{1}_{[0, s]}(\cdot)\right\|_{\kappa, T}^{2} .
$$

We now compare this result to the classical Brownian case with its Markov property.

Remark 3.1. (a) The variance formula above corresponds to

$$
\operatorname{var}\left[X(t) \mid \mathcal{F}_{s}\right]=\operatorname{var}[X(t)]-\operatorname{var}\left[\mathbb{E}\left[X(t) \mid \mathcal{F}_{s}\right]\right]
$$

for $X(t)=\int_{0}^{t} c(v) \mathrm{d} B^{\kappa}(v)$.

(b) By Theorem 3.1 we obtain, for the conditional characteristic function of an FBM,

$$
\begin{aligned}
\mathbb{E}\left[\mathrm{e}^{\mathrm{i} u B^{\kappa}(t)} \mid \mathcal{F}_{s}\right]= & \exp \left\{\mathrm{i} u\left[B^{\kappa}(s)+\int_{0}^{s} \Psi^{\kappa}(s, t, v) \mathrm{d} B^{\kappa}(v)\right]\right\} \\
& \times \exp \left\{-\frac{u^{2}}{2}\left[\left\|\mathbf{1}_{[s, t]}(\cdot)\right\|_{\kappa, T}^{2}-\left\|\Psi^{\kappa}(s, t, \cdot) \mathbf{1}_{[0, s]}(\cdot)\right\|_{\kappa, T}^{2}\right]\right\}, \quad u \in \mathbb{R} .
\end{aligned}
$$

If we compare this to the standard Brownian motion case, i.e. setting $\kappa=0$, we obtain

$$
\mathbb{E}\left[\mathrm{e}^{\mathrm{i} u B^{0}(t)} \mid \mathcal{F}_{s}\right]=\exp \left\{\mathrm{i} u B^{0}(s)-\frac{u^{2}}{2}\left\|\mathbf{1}_{[s, t]}(\cdot)\right\|_{2}^{2}\right\}, \quad u \in \mathbb{R} .
$$

It is not surprising that, for $\kappa \neq 0$, the whole past path plays a role in the prediction. Theorem 3.1 and the equations above show that the conditional expectation changes by the term $\int_{0}^{s} \Psi^{\kappa}(s, t, v) \mathrm{d} B^{\kappa}(v)$.

Consider now a general pathwise SDE with fractional Brownian noise, i.e.

$$
\mathrm{d} Z(t)=\mu(Z(t)) \mathrm{d} t+\sigma(Z(t)) \mathrm{d} B^{\kappa}(t), \quad Z(0) \in \mathbb{R}, t \in[0, T],
$$

for suitable coefficient functions $\mu(\cdot)$ and $\sigma(\cdot)$. Buchmann and Klüppelberg [4] have shown that, for $\kappa \in\left(0, \frac{1}{2}\right)$, and under certain technical conditions on $\mu(\cdot)$ and $\sigma(\cdot)$ (precisely stated in Definition 3.2 of [4]), solutions to (3.1) are given by

$$
\begin{gathered}
Z(t)=f(X(t)), \\
\mathrm{d} X(t)=-a X(t) \mathrm{d} t+\mathrm{d} B^{\kappa}(t), \quad X(0)=f^{-1}(Z(0)), t \in[0, T],
\end{gathered}
$$

for some monotone and differentiable $f: \mathbb{R} \rightarrow \mathbb{R}$ (cf. Definition 3.3 of [4]) and $a>0$. 
Motivated by this, we want to predict general OU-type processes driven by FBM in a next step. Therefore, we consider the SDE with time-dependent coefficient functions, i.e.

$$
\mathrm{d} X(t)=(k(t)-a(t) X(t)) \mathrm{d} t+\sigma(t) \mathrm{d} B^{\kappa}(t), \quad X(0) \in \mathbb{R}, t \in[0, T],
$$

where integration is defined in Section 2. Here $k(\cdot)$ and $a(\cdot)$ are locally integrable and continuous on $\mathbb{R}_{+}$, and $\sigma(\cdot) \neq 0$, is continuous, and belongs to $\Lambda_{T}^{\kappa}$. Assume further that $\exp \left\{-\int_{.}^{t} a(w) \mathrm{d} w\right\} \sigma(\cdot) \in \Lambda_{T}^{\kappa}$ for $0 \leq t \leq T$. Then the unique solution to (3.4) is given by the process $X=(X(t))_{t \in[0, T]}$, defined by

$$
\begin{aligned}
X(t)= & X(0) \exp \left\{-\int_{0}^{t} a(s) \mathrm{d} s\right\}+\int_{0}^{t} \exp \left\{-\int_{s}^{t} a(u) \mathrm{d} u\right\} k(s) \mathrm{d} s \\
& +\int_{0}^{t} \exp \left\{-\int_{s}^{t} a(u) \mathrm{d} u\right\} \sigma(s) \mathrm{d} B^{\kappa}(s), \quad t \in[0, T] .
\end{aligned}
$$

Because $\sigma$ does not hit 0 , we have the equality $\mathscr{F}_{s}=\overline{\sigma\{X(v), v \in[0, s]\}}$ for $0 \leq s \leq T$.

Theorem 3.2. Let $0 \leq s \leq t \leq T$. Set $c(\cdot)=\exp \left\{-\int_{.}^{t} a(w) \mathrm{d} w\right\} \sigma(\cdot)$ and recall $\Psi_{c}^{\kappa}$ from (2.2). Then $X(t) \mid \mathcal{F}_{s}$ is normally distributed with

$$
\begin{aligned}
\mathbb{E}\left[X(t) \mid \mathcal{F}_{s}\right]= & X(s) \exp \left\{-\int_{s}^{t} a(v) \mathrm{d} v\right\}+\int_{s}^{t} \exp \left\{-\int_{v}^{t} a(w) \mathrm{d} w\right\} k(v) \mathrm{d} v \\
& +\int_{0}^{s} \Psi_{c}^{\kappa}(s, t, v) \mathrm{d} B^{\kappa}(v)
\end{aligned}
$$

and

$$
\operatorname{var}\left[X(t) \mid \mathcal{F}_{s}\right]=\left\|c(\cdot) \mathbf{1}_{[s, t]}(\cdot)\right\|_{\kappa, T}^{2}-\left\|\Psi_{c}^{\kappa}(s, t, \cdot) \mathbf{1}_{[0, s]}(\cdot)\right\|_{\kappa, T}^{2} .
$$

If we assume further that $\sigma(\cdot)$ and $1 / \sigma(\cdot)$ are of bounded $p$-variation for some $0<p<$ $1 /\left(\frac{1}{2}-\kappa\right)$, cf. [21], then (3.4) can be considered as a pathwise SDE and, furthermore, the FBMdriven integral in (3.5) exists as the pathwise limit of Riemann-Stieltjes sums (see Section 10 of [21]). An advantage of these stronger assumptions on $\sigma(\cdot)$ is that we are now able to invert SDE (3.5) (since a density formula like that in Theorem A.4 of [10] is needed for this step) and rewrite the prediction in terms of $X$.

Proposition 3.1. In the situation of Theorem 3.2 assume that $\sigma(\cdot)$ and $1 / \sigma(\cdot)$ are of bounded p-variation for some $0<p<1 /\left(\frac{1}{2}-\kappa\right)$. Let $0 \leq s \leq t \leq T$. Set $c(\cdot)=$ $\exp \left\{-\int_{\text {. }}^{t} a(w) \mathrm{d} w\right\} \sigma(\cdot)$ and recall $\Psi_{c}^{\kappa}$ from (2.2). Then $X(t) \mid \mathcal{F}_{S}$ is normally distributed with

$$
\begin{aligned}
\mathbb{E}\left[X(t) \mid \mathcal{F}_{s}\right]= & X(s) \exp \left\{-\int_{s}^{t} a(v) \mathrm{d} v\right\}+\int_{s}^{t} \exp \left\{-\int_{v}^{t} a(w) \mathrm{d} w\right\} k(v) \mathrm{d} v \\
& -\int_{0}^{s} \Psi_{c}^{\kappa}(s, t, v) \frac{k(v)}{\sigma(v)} \mathrm{d} v+\int_{0}^{s} \Psi_{c}^{\kappa}(s, t, v) \frac{a(v)}{\sigma(v)} X(v) \mathrm{d} v \\
& +\int_{0}^{s} \Psi_{c}^{\kappa}(s, t, v) \frac{1}{\sigma(v)} \mathrm{d} X(v)
\end{aligned}
$$

and

$$
\operatorname{var}\left[X(t) \mid \mathcal{F}_{s}\right]=\left\|c(\cdot) \mathbf{1}_{[s, t]}(\cdot)\right\|_{\kappa, T}^{2}-\left\|\Psi_{c}^{\kappa}(s, t, \cdot) \mathbf{1}_{[0, s]}(\cdot)\right\|_{\kappa, T}^{2},
$$

where $c(\cdot)=\exp \left\{-\int_{.}^{t} a(v) \mathrm{d} v\right\} \sigma(\cdot)$. 
Equation (3.2) shows that the FOU process with time-independent coefficient functions is important when considering general fractional SDEs.

Corollary 3.1. Consider SDE (3.4) with $k(\cdot)=0, a(\cdot)=a>0$, and $\sigma(\cdot)=1$. Then the solution $X$ is given by

$$
X(t)=X(0) \mathrm{e}^{-a t}+\int_{0}^{t} \mathrm{e}^{-a(t-s)} \mathrm{d} B^{\kappa}(s), \quad t \in[0, T] .
$$

For $0 \leq s \leq t \leq T, X(t) \mid \mathcal{F}_{s}$ is normally distributed with

and

$$
\mathbb{E}\left[X(t) \mid \mathcal{F}_{s}\right]=X(s) \mathrm{e}^{-a(t-s)}+a \int_{0}^{s} \Psi_{c}^{\kappa}(s, t, v) X(v) \mathrm{d} v+\int_{0}^{s} \Psi_{c}^{\kappa}(s, t, v) \mathrm{d} X(v)
$$

$$
\operatorname{var}\left[X(t) \mid \mathcal{F}_{S}\right]=\left\|c(\cdot) \mathbf{1}_{[s, t]}(\cdot)\right\|_{\kappa, T}^{2}-\left\|\Psi_{c}^{\kappa}(s, t, \cdot) \mathbf{1}_{[0, s]}(\cdot)\right\|_{\kappa, T}^{2},
$$

where $c(\cdot)=\mathrm{e}^{-a(t-\cdot)}$.

When calculating prices in a bond market, the situation arises in which it is not the short rate process $r$ that has to be predicted, but the integrated process in (1.1). The next proposition will deal with this situation. For notational convenience, we set

$$
D(\cdot, t)=\int^{t} \exp \left\{-\int^{v} a(w) \mathrm{d} w\right\} \mathrm{d} v, \quad t \in[0, T] .
$$

Proposition 3.2. Denote by $X$ the process given in (3.5), and let $0 \leq s \leq t \leq T$. Set $c(\cdot)=D(\cdot, t) \sigma(\cdot)$, and recall $\Psi_{c}^{\kappa}$ from (2.2). Assume further that $D(\cdot, t) \sigma(\cdot) \in \bar{\Lambda}_{T}^{\kappa}$. Then $\int_{0}^{t} X(v) \mathrm{d} v \mid \mathcal{F}_{s}$ is normally distributed with

$$
\begin{aligned}
\mathbb{E}\left[\int_{0}^{t} X(v) \mathrm{d} v \mid \mathcal{F}_{s}\right]= & \int_{0}^{s} X(v) \mathrm{d} v+D(s, t) X(s)+\int_{s}^{t} D(v, t) k(v) \mathrm{d} v \\
& +\int_{0}^{s} \Psi_{c}^{\kappa}(s, t, v) \mathrm{d} B^{\kappa}(v)
\end{aligned}
$$

and

$$
\operatorname{var}\left[\int_{0}^{t} X(v) \mathrm{d} v \mid \mathcal{F}_{s}\right]=\left\|c(\cdot) \mathbf{1}_{[s, t]}(\cdot)\right\|_{\kappa, T}^{2}-\left\|\Psi_{c}^{\kappa}(s, t, \cdot) \mathbf{1}_{[0, s]}(\cdot)\right\|_{\kappa, T}^{2} .
$$

If we assume further that $\sigma(\cdot)$ and $1 / \sigma(\cdot)$ are of bounded $p$-variation for some $0<p<$ $1 /\left(\frac{1}{2}-\kappa\right)$, then we have

$$
\begin{aligned}
\mathbb{E}\left[\int_{0}^{t} X(v) \mathrm{d} v \mid \mathcal{F}_{s}\right]= & \int_{0}^{s} X(v) \mathrm{d} v+D(s, t) X(s)+\int_{s}^{t} D(v, t) k(v) \mathrm{d} v \\
& -\int_{0}^{s} \Psi_{c}^{\kappa}(s, t, v) \frac{k(v)}{\sigma(v)} \mathrm{d} v+\int_{0}^{s} \Psi_{c}^{\kappa}(s, t, v) \frac{a(v)}{\sigma(v)} X(v) \mathrm{d} v \\
& +\int_{0}^{s} \Psi_{c}^{\kappa}(s, t, v) \frac{1}{\sigma(v)} \mathrm{d} X(v)
\end{aligned}
$$

and

$$
\operatorname{var}\left[\int_{0}^{t} X(v) \mathrm{d} v \mid \mathcal{F}_{s}\right]=\left\|c(\cdot) \mathbf{1}_{[s, t]}(\cdot)\right\|_{\kappa, T}^{2}-\left\|\Psi_{c}^{\kappa}(s, t, \cdot) \mathbf{1}_{[0, s]}(\cdot)\right\|_{\kappa, T}^{2} .
$$


The next theorem presents the conditional characteristic function of processes $f \circ X$ as in (3.2). In general, $Z$ is no longer Gaussian. Note, however, that because of the assumptions on $f$, we have $\mathcal{F}_{s}=\sigma \overline{\{Z(v), v \in[0, s]\}}$ for $0 \leq s \leq T$.

Theorem 3.3. Let the process $Z$ be given by (3.2) with FOU $X$ as in (3.3) and $0 \leq s \leq t \leq T$. Then we have, for $u \in \mathbb{R}$,

$$
\mathbb{E}\left[\mathrm{e}^{\mathrm{i} u Z(t)} \mid \mathcal{F}_{S}\right]=\int_{\mathbb{R}}\left(\mathbb{E}\left[\mathrm{e}^{(\mathrm{i} \xi+1) X(t)} \mid \mathcal{F}_{S}\right] \widehat{g_{+}}(\xi, u)+\mathbb{E}\left[\mathrm{e}^{(\mathrm{i} \xi-1) X(t)} \mid \mathcal{F}_{S}\right] \widehat{g_{-}}(\xi, u)\right) \mathrm{d} \xi
$$

with

$$
\widehat{g}_{ \pm}(\xi, u)=(2 \pi)^{-1} \int_{\mathbb{R}_{ \pm}} \mathrm{e}^{-(\mathrm{i} \xi \pm 1) x+\mathrm{i} u f(x)} \mathrm{d} x
$$

and $\mathbb{E}\left[\mathrm{e}^{(\mathrm{i} \xi+1) X(t)} \mid \mathcal{F}_{S}\right]$ given by the continuation of the characteristic function of $X(t) \mid \mathcal{F}_{S}$ to $\mathbb{C}$. This continuation exists due to the fact that $X(t) \mid \mathcal{F}_{s}$ is Gaussian.

Since the process $Z$ as given in (3.2) does not have to be Gaussian any longer, there is no closed form for the prediction. However, by Theorem 3.3 we can reduce this problem to an improper integral and the prediction of FOU-type processes.

Example 3.1. (FCIR process.) We consider, for $\kappa \in\left(0, \frac{1}{2}\right)$, an FCIR model given by the pathwise solution to the SDE

$$
\mathrm{d} Z(t)=-\lambda Z(t) \mathrm{d} t+\sigma \sqrt{|Z(t)|} \mathrm{d} B^{\kappa}(t), \quad Z(0) \in \mathbb{R}, t \in[0, T],
$$

for some $\lambda, \sigma>0$. Then by Proposition 5.7 of [4] we know that a solution is given by

$$
\begin{gathered}
Z(t)=f(X(t)) \\
\mathrm{d} X(t)=-\frac{\lambda}{2} X(t) \mathrm{d} t+\mathrm{d} B^{\kappa}(t), \quad X(0)=f^{-1}(Z(0)), t \in[0, T]
\end{gathered}
$$

where $f(x)=\operatorname{sgn}(x) \sigma^{2} x^{2} / 4$. We want to emphasize that this solution, in contrast to the classical Brownian case $(\kappa=0)$, is not unique; for further details, we refer the reader to [10, Proposition 5.1], where a similar case for the more general class of fractional Lévy-driven processes is considered.

In the next section we consider an application of our results to bond markets. Recall that in many cases characteristic functions can be extended from arguments in $\mathbb{R}$ to $\mathbb{C}$.

\section{Application: fractional bond market}

We apply our formulae to calculate the price of a zero-coupon bond, when the short rate is modeled by a fractional Vasicek model with any number of factors. As explained in the introduction, there is empirical evidence of long-range dependence in the bond market. As we also allow for $\kappa=0$, the Brownian case is included.

Similar to the setup of Section 3 of Biagini et al. [3] we can directly model under a riskneutral measure. This is justified by the following argument. As in the classical HJM approach of Heath et al. [14], a fractional Vasicek model can be derived from the fractional HJM model of Ohashi [17]. First the whole forward curve is described under a measure $\mathcal{P}$. Under proportional 
transaction costs with proportionality factor $k>0$, arbitrage can be ruled out and an equivalent measure $\mathcal{Q}$ exists under which bond prices are basically given as conditional expectations.

Ohashi's considerations on a fractional HJM bond model with proportional transaction costs are based on an extension of the full support property of the logarithmic price processes in the set of continuous functions. The basic idea of taking the path properties into account, and their relevance to the absence of arbitrage, was fully investigated by Guasoni et al. [12]. However, these properties are only sufficient for the market to be arbitrage free. A fundamental theorem with necessary and sufficient conditions for risk-neutral asset pricing under proportional transaction costs has been proven by Guasoni et al. [13].

Following Section 3 of [3], let the bond market be described by the short rate process $r=(r(t))_{t \geq 0}$ on the probability space $(\Omega, \mathcal{F}, \mathcal{Q})$, endowed with the filtration $\left(\mathcal{F}_{t}\right)_{t \geq 0}$ generated by $r$, representing the complete market information and satisfying the usual conditions of completeness and right continuity.

We are aware that, as a Gaussian process, the short rate $r$ can also take negative values. However, it is always possible to shift and, perhaps, also scale the model such that the probability of becoming negative is arbitrarily small. On the other hand, it is attractive and useful to have a benchmark model where quantities can be calculated explicitly.

Remaining in this framework and given a maturity date $T>0$, we consider a multivariate FBM given by

$$
\boldsymbol{B}^{\kappa}(t)=\left(B_{(1)}^{\kappa(1)}(t), \ldots, B_{(d)}^{\kappa(d)}(t)\right)^{\top}, \quad t \in[0, T],
$$

for some $d \in \mathbb{N}$ and $\kappa \in\left(-\frac{1}{2}, \frac{1}{2}\right)^{d}$. The components $B_{(i)}^{\kappa(i)}$ for $i=1 \ldots, d$ are assumed to be independent. We remark that, although empirical evidence shows long-range dependence in short rates, our calculations also include the case $\kappa(i) \in\left(-\frac{1}{2}, 0\right)$.

Consider, for

$$
\boldsymbol{X}(0)=\left(X^{(1)}(0), \ldots, X^{(n)}(0)\right)^{\top} \in \mathbb{R}^{d},
$$

a system of $d$ fractional Vasicek SDEs given for $i=1, \ldots, d$ by

$$
\mathrm{d} X^{(i)}(t)=\left(k^{(i)}(t)-a^{(i)}(t) X^{(i)}(t)\right) \mathrm{d} t+\sigma^{(i)}(t) \mathrm{d} B_{(i)}^{\kappa(i)}(t), \quad t \in[0, T] .
$$

We assume that $k^{(i)}(\cdot)$ and $a^{(i)}(\cdot)$ are locally integrable and continuous on $\mathbb{R}_{+}$, and that $\sigma^{(i)}(\cdot) \neq 0$, is continuous, and belongs to $\Lambda_{T}^{\kappa(i)}$. Assume further that $D^{(i)}(\cdot, t) \sigma^{(i)}(\cdot) \in \Lambda_{T}^{\kappa(i)}$ for $0 \leq t \leq T$. Furthermore, let $\sigma^{(i)}(\cdot)$ and $1 / \sigma^{(i)}(\cdot)$ be of bounded $p(i)$-variation for some $0<p(i)<1 /(1-\kappa(i))$. Considering (3.4), the unique solution of (4.1) is given by $\boldsymbol{X}(t)=\left(X^{(1)}(t), \ldots, X^{(d)}(t)\right)^{\top}$, where $X^{(i)}$ is defined as in (3.5).

Now, for fixed $\boldsymbol{b} \in \mathbb{R}^{d}$, define, for $t \in[0, T]$,

$$
r(t)=\boldsymbol{b}^{\top} \boldsymbol{X}(t)
$$

Then $\mathcal{F}_{s}=\sigma \overline{\{r(v), v \in[0, s]\}}$ for $0 \leq s \leq T$. The price of a zero-coupon bond for a short rate given in (4.2) is calculated in the next theorem.

Theorem 4.1. Assume that the situation above holds, and let $0 \leq s \leq t \leq T$. For $i=1, \ldots, d$, set $c_{i}(\cdot)=D^{(i)}(\cdot, t) \sigma^{(i)}(\cdot)$ with $D$ defined in (3.6), and recall $\Psi_{c}^{\kappa}$ from (2.2). Assume further that $D^{(i)}(\cdot, t) \sigma^{(i)}(\cdot) \in \Lambda_{T}^{\kappa(i)}$. Then the price of a zero-coupon bond $B(s, t)$ at time $s$ with 
maturity $t$ is given by

$$
\begin{aligned}
B(s, t)= & \mathbb{E}\left[\exp \left\{-\int_{s}^{t} r(v) \mathrm{d} v\right\} \mid \mathcal{F}_{s}\right] \\
= & \prod_{i=1}^{d} \exp \left\{-b^{(i)}\left[D^{(i)}(s, t) X^{(i)}(s)+\int_{s}^{t} D^{(i)}(v, t) k^{(i)}(v) \mathrm{d} v\right.\right. \\
& \quad-\int_{0}^{s} \Psi_{c^{(i)}}^{\kappa(i)}(s, t, v) \frac{k^{(i)}(v)}{\sigma^{(i)}(v)} \mathrm{d} v+\int_{0}^{s} \Psi_{c^{(i)}}^{\kappa(i)}(s, t, v) \frac{a^{(i)}(v)}{\sigma^{(i)}(v)} X^{(i)}(v) \mathrm{d} v \\
& \left.\left.\quad+\int_{0}^{s} \Psi_{c^{(i)}}^{\kappa(i)}(s, t, v) \frac{1}{\sigma^{(i)}(v)} \mathrm{d} X^{(i)}(v)\right]\right\} \\
& \times \exp \left\{\frac{\left(b^{(i)}\right)^{2}}{2}\left[\left\|c^{(i)}(\cdot) \mathbf{1}_{[s, t]}(\cdot)\right\|_{\kappa^{(i), T}}^{2}-\left\|\Psi_{c^{(i)}}^{\kappa(i)}(s, t, \cdot) \mathbf{1}_{[0, s]}(\cdot)\right\|_{\kappa(i), T}^{2}\right]\right\} .
\end{aligned}
$$

Proof. We calculate

$$
\begin{aligned}
B(s, t) & =\mathbb{E}\left[\exp \left\{-\int_{s}^{t} r(v) \mathrm{d} v\right\} \mid \mathcal{F}_{s}\right] \\
& =\mathbb{E}\left[\exp \left\{-\int_{s}^{t} \boldsymbol{b}^{\top} \boldsymbol{X}(v) \mathrm{d} v\right\} \mid \mathcal{F}_{s}\right] \\
& =\prod_{i=1}^{d} \mathbb{E}\left[\exp \left\{\int_{s}^{t} b^{(i)} X^{(i)}(v) \mathrm{d} v\right\} \mid \mathcal{F}_{s}\right],
\end{aligned}
$$

where we have used the independence of the $X^{(i)}$ in the last equality. The result now follows by an application of Proposition 3.2. The extension of the conditional characteristic function to the whole of the complex plane $\mathbb{C}$ exists because of Gaussianity.

Example 4.1. (Fractional one-factor model.) We want to compare prices in our fractional model to the classical Brownian case, i.e. $\kappa=0$. For simplicity, we assume constant coefficient functions in (4.1) and set $d=1$ with $b=1$. Today's prices of the zero-coupon bonds are given by

$$
B(0, t)=\exp \left\{-D(0, t) X(0)-k \int_{0}^{t} D(v, t) \mathrm{d} v+\frac{\sigma^{2}}{2}\left\|D(\cdot, t) \mathbf{1}_{[0, t]}(\cdot)\right\|_{\kappa, T}^{2}\right\}, \quad t \geq 0 .
$$

Since negative $\kappa$ is not relevant, as explained before, we consider only $\kappa \in\left[0, \frac{1}{2}\right)$. Standard numerical methods may be unstable here because of the singularities in the norms in (4.4) whose exact values cannot be computed. Therefore, we apply the following discretization scheme for $\kappa \in\left(0, \frac{1}{2}\right)$ and $t \in[0, T]$. We have

$$
\begin{aligned}
\left\|D(\cdot, t) \mathbf{1}_{[0, t]}(\cdot)\right\|_{\kappa, T}^{2}= & \frac{\pi \kappa(2 \kappa+1)}{\Gamma(1-2 \kappa) \sin (\pi \kappa)(\Gamma(\kappa))^{2}} \\
& \times \int_{0}^{T} s^{-2 \kappa}\left(\int_{s}^{T} \frac{r^{\kappa} D(r, t) \mathbf{1}_{[0, t]}(r)}{(r-s)^{1-\kappa}} \mathrm{d} r\right)^{2} \mathrm{~d} s .
\end{aligned}
$$


In a first step we decompose the outer integral for $n \in \mathbb{N}$ and $0=s_{0} \leq s_{1} \leq \cdots \leq s_{n}=T$ :

$$
\begin{aligned}
\int_{0}^{T} & s^{-2 \kappa}\left(\int_{s}^{T} \frac{r^{\kappa} D(r, t) \mathbf{1}_{[0, t]}(r)}{(r-s)^{1-\kappa}} \mathrm{d} r\right)^{2} \mathrm{~d} s \\
& =\sum_{i=0}^{n-1} \int_{s_{i}}^{s_{i+1}} s^{-2 \kappa}\left(\int_{s}^{T} \frac{r^{\kappa} D(r, t) \mathbf{1}_{[0, t]}(r)}{(r-s)^{1-\kappa}} \mathrm{d} r\right)^{2} \mathrm{~d} s .
\end{aligned}
$$

For sufficiently small intervals $\left[s_{i}, s_{i+1}\right]$, we get a reasonable approximation by

$$
\int_{s}^{T} \frac{r^{\kappa} D(r, t) \mathbf{1}_{[0, t]}(r)}{(r-s)^{1-\kappa}} \mathrm{d} r \approx \int_{s_{i}}^{T} \frac{r^{\kappa} D(r, t) \mathbf{1}_{[0, t]}(r)}{\left(r-s_{i}\right)^{1-\kappa}} \mathrm{d} r .
$$

Now we take, for $i=0, \ldots, n-1$, a partition $s_{i}=u_{0}^{i} \leq u_{1}^{i} \leq \cdots \leq u_{m_{i}}^{i}=s_{i+1}$ for some $m_{i} \in \mathbb{N}$ :

$$
\begin{aligned}
\int_{s_{i}}^{T} \frac{r^{\kappa} D(r, t) \mathbf{1}_{[0, t]}(r)}{\left(r-s_{i}\right)^{1-\kappa}} \mathrm{d} r \\
\quad=\sum_{j=0}^{m_{i}-1} \int_{u_{j}^{i}}^{u_{j+1}^{i}} \frac{r^{\kappa} D(r, t) \mathbf{1}_{[0, t]}(r)}{\left(r-s_{i}\right)^{1-\kappa}} \mathrm{d} r \\
\quad \approx \frac{1}{\kappa} \sum_{j=0}^{m_{i}-1}\left[\left(u_{j+1}^{i}-s_{i}\right)^{\kappa}-\left(u_{j}^{i}-s_{i}\right)^{\kappa}\right] \frac{\left(u_{j}^{i}\right)^{\kappa} D\left(u_{j}^{i}, t\right)+\left(u_{j+1}^{i}\right)^{\kappa} D\left(u_{j+1}^{i}, t\right)}{2} .
\end{aligned}
$$

Putting everything together and using $\Gamma(\kappa) \cdot \kappa=\Gamma(\kappa+1)$, we obtain

$$
\begin{aligned}
\left\|D(\cdot, t) \mathbf{1}_{[0, t]}(\cdot)\right\|_{\kappa, T}^{2} & \\
\approx & \frac{\pi \kappa(2 \kappa+1)}{\Gamma(2-2 \kappa) \sin (\pi \kappa)(2 \Gamma(\kappa+1))^{2}} \\
& \times \sum_{i=0}^{n-1}\left[s_{i+1}^{1-2 \kappa}-s_{i}^{1-2 \kappa}\right] \\
& \quad \times\left[\sum_{j=0}^{m_{i}-1}\left[\left(u_{j+1}^{i}-s_{i}\right)^{\kappa}-\left(u_{j}^{i}-s_{i}\right)^{\kappa}\right]\left(u_{j}^{i}\right)^{\kappa} D\left(u_{j}^{i}, t\right)+\left(u_{j+1}^{i}\right)^{\kappa} D\left(u_{j+1}^{i}, t\right)\right]^{2} .
\end{aligned}
$$

Choosing $s_{i}=0.01 i$ for $i=0, \ldots, 100 t$, and $u_{j}^{i}=0.01(i+j)$ for $j=0, \ldots, 100 t-i$, we obtain

$$
\begin{aligned}
& \left\|D(\cdot, t) \mathbf{1}_{[0, t]}(\cdot)\right\|_{\kappa, T}^{2} \\
& \approx \frac{\pi \kappa(2 \kappa+1)}{\Gamma(2-2 \kappa) \sin (\pi \kappa) 2 \Gamma(\kappa+1)^{2}} 0.01^{1+2 \kappa} \\
& \quad \times \sum_{i=0}^{100 t-1}\left[(i+1)^{1-2 \kappa}-i^{1-2 \kappa}\right] \\
& \quad \times\left[\sum _ { j = 0 } ^ { 1 0 0 t - i - 1 } [ ( j + 1 ) ^ { \kappa } - j ^ { \kappa } ] \left[(i+j)^{\kappa} D(0.01(i+j), t)\right.\right. \\
& \left.\left.\quad+(i+j+1)^{\kappa} D(0.01(i+j+1), t)\right]\right]^{2} .
\end{aligned}
$$

Examples of the norms and bond prices can be found in Figures 1 and 2 . 


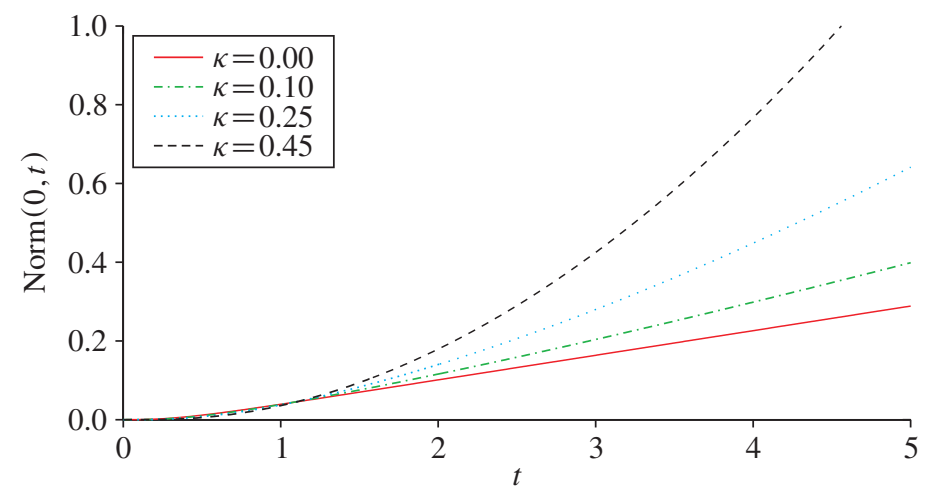

Figure 1: Calculation of $\left\|D(\cdot, t) \mathbf{1}_{[0, t]}(\cdot)\right\|_{\kappa, T}^{2}$ in the fractional one-factor model for varying $\kappa$ and maturity $t$, using $a=4$. The case $\kappa=0$ has been calculated analytically.

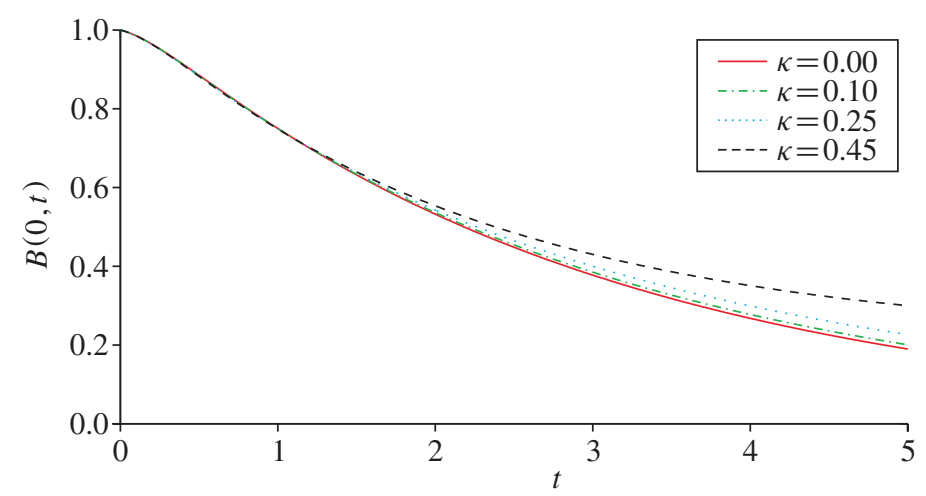

FiguRE 2: Bond prices $B(0, t)$ in the fractional one-factor Vasicek model (4.3) for varying $\kappa \geq 0$ and maturity $t$, using constant coefficients $a=4, k=1$, and $\sigma=1$. Negative $\kappa$ is not relevant, as explained in the introduction of Section 4. Recall that $\kappa=0$ corresponds to the Brownian Vasicek model. Prices increase with $\kappa$ as a consequence of long-range dependence.

\section{Proofs}

There are different ways of proving Theorem 3.1; one possibility is to use Remark 3.1(a) for $X=\int_{s}^{t} c(v) \mathrm{d} B^{\kappa}(v)$. However, having discrete observations in mind (as is the realistic statistical setup), we base our proof on a discretization scheme for the past. Given the grid size and also $\kappa$, we obtain not only a limit result, but also an approximation based on the correct approximating matrices $\left(\Sigma_{22}^{n}\right)^{-1} \Sigma_{21}^{n}$ and $\Sigma_{12}^{n}\left(\Sigma_{22}^{n}\right)^{-1} \Sigma_{21}^{n}$.

For this discrete approximation, we will need the well-known property of the multivariate normal distribution. For $d \in \mathbb{N}$, let $\mathbb{S}^{d \times d}$ denote the space of all positive semidefinite symmetric matrices of dimension $d$.

Lemma 5.1. Let $Z \sim N(\mu, \Sigma)$, i.e. $Z=\left(z_{1}, \ldots, z_{d}\right)^{\top}$ is multivariate normally distributed with mean $\mu \in \mathbb{R}^{d}$ and variance-covariance matrix $\Sigma \in \mathbb{S}^{d \times d}$. For $k \in\{1, \ldots, d-1\}$, set $X=\left(z_{1}, \ldots, z_{k}\right)^{\top}$ and $Y=\left(z_{k+1}, \ldots, z_{d}\right)^{\top}$. Partition

$$
\mu=\left(\begin{array}{l}
\mu_{1} \\
\mu_{2}
\end{array}\right) \text { and } \Sigma=\left(\begin{array}{cc}
\Sigma_{11} & \Sigma_{12} \\
\Sigma_{21} & \Sigma_{22}
\end{array}\right)
$$

with $\mu_{1} \in \mathbb{R}^{k}, \quad \mu_{2} \in \mathbb{R}^{d-k}, \quad \Sigma_{11} \in \mathbb{S}^{k \times k}, \quad \Sigma_{22} \in \mathbb{S}^{(d-k) \times(d-k)}$, and $\Sigma_{12}^{\top}=\Sigma_{21} \in \mathbb{R}^{(d-k) \times k}$. 
Then we have

$$
X \mid\{Y=y\} \sim N\left(\mu_{1}+\Sigma_{12} \Sigma_{22}^{-1}\left(y-\mu_{2}\right), \Sigma_{11}-\Sigma_{12} \Sigma_{22}^{-1} \Sigma_{21}\right) .
$$

Now we can start with the proofs of our results.

Proof of Theorem 3.1. Let $0 \leq s \leq t \leq T$. To calculate the conditional characteristic function of $\int_{0}^{t} c(v) \mathrm{d} B^{\kappa}(v)$, we invoke the fact that, by Gaussianity and Lemma 5.1, the conditional random variable $\int_{0}^{t} c(v) \mathrm{d} B^{\kappa}(v) \mid \mathcal{F}_{s}$ is again normally distributed. Since $\int_{0}^{s} c(v) \mathrm{d} B^{\kappa}(v)$ is $\tilde{F}_{s}$-measurable, it suffices to consider $\int_{s}^{t} c(v) \mathrm{d} B^{\kappa}(v) \mid \mathcal{F}_{s}$.

From Proposition 2.1 we know that

$$
\mathbb{E}\left[\int_{s}^{t} c(v) \mathrm{d} B^{\kappa}(v) \mid \mathcal{F}_{s}\right]=\int_{0}^{s} \Psi_{c}^{\kappa}(s, t, v) \mathrm{d} B^{\kappa}(v),
$$

and, therefore, we need to calculate only the conditional variance $\operatorname{var}\left[\int_{s}^{t} c(v) \mathrm{d} B^{\kappa}(v) \mid \mathcal{F}_{S}\right]$.

Choose a sequence of partitions $\left(\pi_{n}\right)_{n \in \mathbb{N}}$ of $[0, s]$ such that, for $n \in \mathbb{N}$, we have $\pi_{n}=$ $\left(s_{i}^{n}\right)_{i=0, \ldots, m_{n}}$ for $m_{n} \in \mathbb{N}$ with

$$
0=s_{0}^{n}<s_{1}^{n}<\cdots<s_{m_{n}}^{n} \leq s \quad \text { and } \quad \sup _{i=1, \ldots, m_{n}}\left|s_{i}^{n}-s_{i-1}^{n}\right| \rightarrow 0 \quad \text { as } n \rightarrow \infty .
$$

Using this notation, we know from Lemma 5.1 that, for $n \in \mathbb{N}$,

$$
\begin{gathered}
\mathbb{E}\left[\int_{s}^{t} c(v) \mathrm{d} B^{\kappa}(v) \mid \begin{array}{c}
\left.B^{\kappa}\left(s_{i}^{n}\right)-B^{\kappa}\left(s_{i-1}^{n}\right), i=1, \ldots, m_{n}\right] \\
\vdots \\
=\Sigma_{12}^{n}\left(\Sigma_{22}^{n}\right)^{-1}\left(\begin{array}{c}
B^{\kappa}\left(s_{i}^{n}\right)-B^{\kappa}\left(s_{i-1}^{n}\right) \\
\vdots
\end{array}\right)
\end{array}\right.
\end{gathered}
$$

and

$$
\begin{aligned}
& \operatorname{var}\left[\int_{s}^{t} c(v) \mathrm{d} B^{\kappa}(v) \mid B^{\kappa}\left(s_{i}^{n}\right)-B^{\kappa}\left(s_{i-1}^{n}\right), i=1, \ldots, m_{n}\right] \\
& \quad=\Sigma_{11}^{n}-\Sigma_{12}^{n}\left(\Sigma_{22}^{n}\right)^{-1} \Sigma_{21}^{n},
\end{aligned}
$$

where $\Sigma_{11}^{n}=\operatorname{var}\left[\int_{s}^{t} c(v) \mathrm{d} B^{\kappa}(v)\right]$,

$$
\begin{gathered}
\left(\Sigma_{12}^{n}\right)^{\top}=\Sigma_{21}^{n}=\left(\begin{array}{c}
\operatorname{cov}\left[\int_{s}^{t} c(v) \mathrm{d} B^{\kappa}(v), B^{\kappa}\left(s_{i}^{n}\right)-B^{\kappa}\left(s_{i-1}^{n}\right)\right] \\
\vdots
\end{array}\right) \in \mathbb{R}^{m_{n}}, \\
\Sigma_{22}^{n}=\left(\operatorname{cov}\left[B^{\kappa}\left(s_{i}^{n}\right)-B^{\kappa}\left(s_{i-1}^{n}\right), B^{\kappa}\left(s_{j}^{n}\right)-B^{\kappa}\left(s_{j-1}^{n}\right)\right]\right)_{i, j=1, \ldots, m_{n}} \in \mathbb{S}^{m_{n} \times m_{n}} .
\end{gathered}
$$

It follows from Lemma 5.1 and [5, p. 290] that, almost surely (a.s.) as $n \rightarrow \infty$,

$$
\mathbb{E}\left[\int_{s}^{t} c(v) \mathrm{d} B^{\kappa}(v) \mid B^{\kappa}\left(s_{i}^{n}\right)-B^{\kappa}\left(s_{i-1}^{n}\right), i=1, \ldots, m_{n}\right] \rightarrow \mathbb{E}\left[\int_{s}^{t} c(v) \mathrm{d} B^{\kappa}(v) \mid \mathcal{F}_{s}\right]
$$

and

$$
\operatorname{var}\left[\int_{s}^{t} c(v) \mathrm{d} B^{\kappa}(v) \mid B^{\kappa}\left(s_{i}^{n}\right)-B^{\kappa}\left(s_{i-1}^{n}\right), i=1, \ldots, m_{n}\right] \rightarrow \operatorname{var}\left[\int_{s}^{t} c(v) \mathrm{d} B^{\kappa}(v) \mid \mathcal{F}_{S}\right] .
$$


It then follows by (5.1) and Proposition 2.1 that, a.s. as $n \rightarrow \infty$,

$$
\begin{aligned}
\Sigma_{12}^{n}\left(\Sigma_{22}^{n}\right)^{-1}\left(\begin{array}{c}
B^{\kappa}\left(s_{i}^{n}\right)-B^{\kappa}\left(s_{i-1}^{n}\right) \\
\vdots
\end{array}\right) & =\sum_{i=1}^{m_{n}}\left(\Sigma_{12}^{n}\left(\Sigma_{22}^{n}\right)^{-1}\right)_{i}\left[B^{\kappa}\left(s_{i}^{n}\right)-B^{\kappa}\left(s_{i-1}^{n}\right)\right] \\
& \rightarrow \int_{0}^{s} \Psi_{c}^{\kappa}(s, t, v) \mathrm{d} B^{\kappa}(v) .
\end{aligned}
$$

Therefore, in $\|\cdot\|_{\kappa, T}$ as $n \rightarrow \infty$,

$$
\sum_{i=1}^{m_{n}}\left(\Sigma_{12}^{n}\left(\Sigma_{22}^{n}\right)^{-1}\right)_{i} \mathbf{1}_{\left[s_{i-1}^{n}, s_{i}^{n}\right]}(\cdot) \rightarrow \Psi_{c}^{\kappa}(s, t, \cdot) \mathbf{1}_{[0, s]}(\cdot) .
$$

With this result we can now calculate the conditional variance, since, using isometry (2.1),

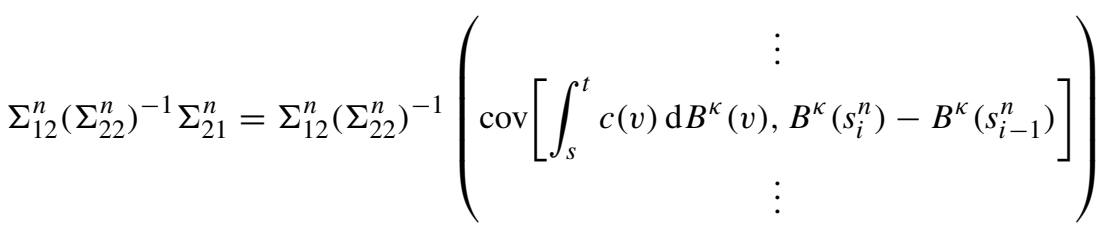

$$
\begin{aligned}
& =\sum_{i=1}^{m_{n}}\left(\Sigma_{12}^{n}\left(\Sigma_{22}^{n}\right)^{-1}\right)_{i} \operatorname{cov}\left[\int_{s}^{t} c(v) \mathrm{d} B^{\kappa}(v), B^{\kappa}\left(s_{i}^{n}\right)-B^{\kappa}\left(s_{i-1}^{n}\right)\right] \\
& =\sum_{i=1}^{m_{n}}\left(\Sigma_{12}^{n}\left(\Sigma_{22}^{n}\right)^{-1}\right)_{i}\left\langle c(\cdot) \mathbf{1}_{[0, s]}(\cdot), \mathbf{1}_{\left[s_{i-1}^{n}, s_{i}^{n}\right]}(\cdot)\right\rangle_{\kappa, T} \\
& =\left\langle c(\cdot) \mathbf{1}_{[0, s]}(\cdot), \sum_{i=1}^{m_{n}}\left(\Sigma_{12}^{n}\left(\Sigma_{22}^{n}\right)^{-1}\right)_{i} \mathbf{1}_{\left[s_{i-1}^{n}, s_{i}^{n}\right]}(\cdot)\right\rangle_{\kappa, T} \\
& \rightarrow\left\langle c(\cdot) \mathbf{1}_{[0, s]}(\cdot), \Psi_{c}^{\kappa}(s, t, \cdot) \mathbf{1}_{[0, s]}(\cdot)\right\rangle_{\kappa, T} \quad \text { as } n \rightarrow \infty,
\end{aligned}
$$

where in the last line we have used the continuity of the scalar product.

It remains to observe that, again by isometry (2.1),

$$
\begin{aligned}
\left\langle c(\cdot) \mathbf{1}_{[0, s]}(\cdot), \Psi_{c}^{\kappa}(s, t, \cdot) \mathbf{1}_{[0, s]}(\cdot)\right\rangle_{\kappa, T} \\
=\mathbb{E}\left[\int_{s}^{t} c(v) \mathrm{d} B^{\kappa}(v) \int_{0}^{s} \Psi_{c}^{\kappa}(s, t, v) \mathrm{d} B^{\kappa}(v)\right] \\
=\mathbb{E}\left[\left(\int_{s}^{t} c(v) \mathrm{d} B^{\kappa}(v)-\mathbb{E}\left[\int_{s}^{t} c(v) \mathrm{d} B^{\kappa}(v) \mid \mathcal{F}_{s}\right]\right) \mathbb{E}\left[\int_{s}^{t} c(v) \mathrm{d} B^{\kappa}(v) \mid \mathcal{F}_{s}\right]\right] \\
\quad+\mathbb{E}\left[\mathbb{E}\left[\int_{s}^{t} c(v) \mathrm{d} B^{\kappa}(v) \mid \mathcal{F}_{s}\right]^{2}\right] \\
=\mathbb{E}\left[\mathbb{E}\left[\int_{s}^{t} c(v) \mathrm{d} B^{\kappa}(v) \mid \mathcal{F}_{s}\right]^{2}\right] \\
=\left\|\Psi_{c}^{\kappa}(s, t, \cdot) \mathbf{1}_{[0, s]}(\cdot)\right\|_{\kappa, T}^{2}
\end{aligned}
$$

by the projection property of the conditional expectation in $L^{2}$. 
Finally, we conclude that, a.s.,

$$
\begin{aligned}
\operatorname{var}\left[\int_{s}^{t} c(v) \mathrm{d} B^{\kappa}(v) \mid \mathcal{F}_{s}\right] \\
\quad=\lim _{n \rightarrow \infty} \operatorname{var}\left[\int_{s}^{t} c(v) \mathrm{d} B^{\kappa}(v) \mid B^{\kappa}\left(s_{i}^{n}\right)-B^{\kappa}\left(s_{i-1}^{n}\right), i=1, \ldots, m_{n}\right] \\
=\lim _{n \rightarrow \infty}\left(\Sigma_{11}^{n}-\Sigma_{21}^{n}\left(\Sigma_{22}^{n}\right)^{-1} \Sigma_{12}^{n}\right) \\
=\left\|c(\cdot) \mathbf{1}_{[s, t]}(\cdot)\right\|_{\kappa, T}-\left\langle c(\cdot) \mathbf{1}_{[0, s]}(\cdot), \Psi_{c}^{\kappa}(s, t, \cdot) \mathbf{1}_{[0, s]}(\cdot)\right\rangle_{\kappa, T} \\
=\left\|c(\cdot) \mathbf{1}_{[s, t]}(\cdot)\right\|_{\kappa, T}-\left\|\Psi_{c}^{\kappa}(s, t, \cdot) \mathbf{1}_{[0, s]}(\cdot)\right\|_{\kappa, T}^{2} .
\end{aligned}
$$

Theorem 3.2 is now a consequence of Theorem 3.1.

Proof of Theorem 3.2. By (3.5), it follows that, for $0 \leq s \leq t \leq T$,

$$
\begin{aligned}
X(t)= & X(s) \exp \left\{-\int_{s}^{t} a(v) \mathrm{d} v\right\}+\int_{s}^{t} \exp \left\{-\int_{v}^{t} a(w) \mathrm{d} w\right\} k(v) \mathrm{d} v \\
& +\int_{s}^{t} \exp \left\{-\int_{v}^{t} a(w) \mathrm{d} w\right\} \sigma(v) \mathrm{d} B^{\kappa}(v) .
\end{aligned}
$$

Therefore, $X(t) \mid \mathcal{F}_{s}$ is again Gaussian distributed. Since $X(s)$ is $\mathcal{F}_{s}$-measurable, a direct consequence is now that

$$
\begin{aligned}
\mathbb{E}\left[X(t) \mid \mathcal{F}_{s}\right]= & X(s) \exp \left\{-\int_{s}^{t} a(v) \mathrm{d} v\right\}+\int_{s}^{t} \exp \left\{-\int_{v}^{t} a(w) \mathrm{d} w\right\} k(v) \mathrm{d} v \\
& +\mathbb{E}\left[\int_{s}^{t} \exp \left\{-\int_{v}^{t} a(w) \mathrm{d} w\right\} \sigma(v) \mathrm{d} B^{\kappa}(v) \mid \mathcal{F}_{s}\right]
\end{aligned}
$$

and

$$
\operatorname{var}\left[X(t) \mid \mathcal{F}_{S}\right]=\operatorname{var}\left[\int_{s}^{t} \exp \left\{-\int_{v}^{t} a(w) \mathrm{d} w\right\} \sigma(v) \mathrm{d} B^{\kappa}(v) \mid \mathcal{F}_{S}\right]
$$

Invoking Theorem 3.1 with $c(\cdot)=\exp \left\{-\int_{.}^{t} a(w) \mathrm{d} w\right\} \sigma(\cdot)$ concludes the proof.

The main step of the proof of Proposition 3.1 is an application of a density formula for Riemann-Stieltjes integrals.

Proof of Proposition 3.1. By assumption on the coefficient functions, all integrals appearing in this proof can be considered in the pathwise Riemann-Stieltjes sense; cf. [21, Section 10].

Our goal is now to invert (3.4). By (3.5) we have, for $0 \leq s \leq t \leq T$,

$$
\begin{aligned}
& \int_{s}^{t} \exp \left\{-\int_{v}^{t} a(w) \mathrm{d} w\right\} \sigma(v) \mathrm{d} B^{\kappa}(v) \\
& \quad=X(t)-X(s) \exp \left\{-\int_{v}^{t} a(w) \mathrm{d} w\right\}-\int_{s}^{t} \exp \left\{-\int_{v}^{t} a(w) \mathrm{d} w\right\} k(v) \mathrm{d} v
\end{aligned}
$$


and, invoking a density formula (which can be applied using Theorem A.4 of [10]) we obtain

$$
\begin{gathered}
B^{\kappa}(t)-B^{\kappa}(s)=\int_{s}^{t} \frac{\exp \left\{\int_{v}^{t} a(w) \mathrm{d} w\right\}}{\sigma(v)} \mathrm{d}\left(-\int_{v}^{t} \exp \left\{-\int_{z}^{t} a(w) \mathrm{d} w\right\} \sigma(z) \mathrm{d} B^{\kappa}(z)\right) \\
=\int_{s}^{t} \frac{\exp \left\{\int_{v}^{t} a(w) \mathrm{d} w\right\}}{\sigma(v)} \mathrm{d}\left(\int_{v}^{t} \exp \left\{-\int_{z}^{t} a(w) \mathrm{d} w\right\} k(z) \mathrm{d} z\right. \\
\left.\quad+X(v) \exp \left\{-\int_{v}^{t} a(w) \mathrm{d} w\right\}-X(t)\right) \\
=-\int_{s}^{t} \frac{k(v)}{\sigma(v)} \mathrm{d} v+\int_{s}^{t} \frac{a(v)}{\sigma(v)} X(v) \mathrm{d} v+\int_{s}^{t} \frac{1}{\sigma(v)} \mathrm{d} X(v) .
\end{gathered}
$$

To complete the proof, substitute this result into the formulae of Theorem 3.2.

Proof of Proposition 3.2. Let $0 \leq s \leq t \leq T$. By Gaussianity we again see that $\int_{0}^{t} X(v) \mathrm{d} v \mid \mathcal{F}_{S}$ is normally distributed, and, as before, it remains to calculate its expectation and variance to achieve the conditional characteristic function. Since $\int_{0}^{s} X(v) \mathrm{d} v$ is $\mathcal{F}_{s}$-measurable, we just consider $\int_{s}^{t} X(v) \mathrm{d} v \mid \mathcal{F}_{s}$. From (3.5) we obtain, by (5.2) and Fubini's theorem (see Theorem 1 of [16]),

$$
\begin{aligned}
\int_{s}^{t} X(v) \mathrm{d} v= & \int_{s}^{t}\left\{X(s) \exp \left\{-\int_{s}^{v} a(w) \mathrm{d} w\right\}+\int_{s}^{v} \exp \left\{-\int_{z}^{v} a(w) \mathrm{d} w\right\} k(z) \mathrm{d} z\right. \\
& \left.+\int_{s}^{v} \exp \left\{-\int_{z}^{v} a(w) \mathrm{d} w\right\} \sigma(z) \mathrm{d} B^{\kappa}(z)\right\} \mathrm{d} v \\
= & D(s, t) X(s)+\int_{s}^{t} D(v, t) k(v) \mathrm{d} v+\int_{s}^{t} D(v, t) \sigma(v) \mathrm{d} B^{\kappa}(v) .
\end{aligned}
$$

It follows that

$$
\begin{aligned}
\mathbb{E}\left[\int_{0}^{t} X(v) \mathrm{d} v \mid \mathcal{F}_{s}\right]= & \int_{0}^{s} X(v) \mathrm{d} v+D(s, t) X(s)+\int_{s}^{t} D(v, t) k(v) \mathrm{d} v \\
& +\mathbb{E}\left[\int_{s}^{t} D(v, t) \sigma(v) \mathrm{d} B^{\kappa}(v) \mid \mathcal{F}_{s}\right]
\end{aligned}
$$

and

$$
\operatorname{var}\left[\int_{0}^{t} X(v) \mathrm{d} v \mid \mathcal{F}_{S}\right]=\operatorname{var}\left[\int_{s}^{t} D(v, t) \sigma(v) \mathrm{d} B^{\kappa}(v) \mid \mathcal{F}_{S}\right] .
$$

Applying Theorem 3.1 with $c(\cdot)=D(\cdot, t) \sigma(\cdot)$ shows the first assertion. The second assertion follows by applying (5.3).

The proof of Theorem 3.3 uses Fourier techniques.

Proof of Theorem 3.3. Let $x \in \mathbb{R}$, and set $g(x, u)=\exp (\mathrm{i} u f(x))$. First we decompose $g$ as follows:

$$
\begin{aligned}
g(x, u) & =\mathrm{e}^{x}\left[\mathrm{e}^{-x} g(x, u) \mathbf{1}_{[0, \infty)}(x)\right]+\mathrm{e}^{-x}\left[\mathrm{e}^{x} g(x, u) \mathbf{1}_{(-\infty, 0)}(x)\right] \\
& =: \mathrm{e}^{x} g_{+}(x, u)+\mathrm{e}^{-x} g_{-}(x, u) .
\end{aligned}
$$


For fixed $u \in \mathbb{R}$, denote by $\widehat{g}_{+}(\cdot, u)$ and $\widehat{g}_{-}(\cdot, u)$ the Fourier transforms of $g_{+}(\cdot, u)$ and $g_{-}(\cdot, u)$, respectively. Using classical Fourier analysis, we obtain, for $x, \xi \in \mathbb{R}$,

$$
\begin{aligned}
& \widehat{g}_{ \pm}(\xi, u)=\frac{1}{2 \pi} \int_{\mathbb{R}} \mathrm{e}^{-\mathrm{i} \xi x} g_{ \pm}(x, u) \mathrm{d} x=\frac{1}{2 \pi} \int_{\mathbb{R}_{ \pm}} \mathrm{e}^{-(\mathrm{i} \xi \pm 1) x+\mathrm{i} u f(x)} \mathrm{d} x, \\
& g_{ \pm}(x, u)=\int_{\mathbb{R}} \mathrm{e}^{\mathrm{i} \xi x} \widehat{g}_{ \pm}(\xi, u) \mathrm{d} \xi
\end{aligned}
$$

where we have used the fact that $g_{+}(\cdot, u)$ and $g_{-}(\cdot, u)$ are in $L^{1}(\mathbb{R}) \cap L^{2}(\mathbb{R})$ since $g(\cdot, u)$ is bounded. Now we obtain

$$
\begin{aligned}
\mathbb{E}\left[\mathrm{e}^{\mathrm{i} u Z(t)} \mid \mathcal{F}_{s}\right]= & \mathbb{E}\left[g(X(t)) \mid \mathcal{F}_{S}\right] \\
= & \mathbb{E}\left[\mathrm{e}^{X(t)} g_{+}(X(t), u) \mid \mathcal{F}_{S}\right]+\mathbb{E}\left[\mathrm{e}^{-X(t)} g_{-}(X(t), u) \mid \mathcal{F}_{s}\right] \\
= & \mathbb{E}\left[\mathrm{e}^{X(t)} \int_{\mathbb{R}} \mathrm{e}^{\mathrm{i} \xi X(t)} \widehat{g}_{+}(\xi, u) \mathrm{d} \xi \mid \mathcal{F}_{s}\right] \\
& +\mathbb{E}\left[\mathrm{e}^{-X(t)} \int_{\mathbb{R}} \mathrm{e}^{\mathrm{i} \xi X(t)} \widehat{g}_{-}(\xi, u) \mathrm{d} \xi \mid \mathcal{F}_{S}\right] .
\end{aligned}
$$

Since $\mathbb{E}\left[\mathrm{e}^{b X(t)}\right]<\infty$ for all $b \in \mathbb{C}$, we can interchange the conditional expectation and integration to obtain

$$
\mathbb{E}\left[\mathrm{e}^{\mathrm{i} u Z(t)} \mid \mathcal{F}_{S}\right]=\int_{\mathbb{R}}\left(\mathbb{E}\left[\mathrm{e}^{(\mathrm{i} \xi+1) X(t)} \mid \mathcal{F}_{S}\right] \widehat{g}_{+}(\xi, u)+\mathbb{E}\left[\mathrm{e}^{(\mathrm{i} \xi-1) X(t)} \mid \mathcal{F}_{S}\right] \widehat{g}_{-}(\xi, u)\right) \mathrm{d} \xi
$$

\section{References}

[1] BaCKUS, D. K. AND ZiN, S. E. (1993). Long-memory inflation uncertainty: evidence from the term structure of interest rates. J. Money Credit Banking 25, 681-700.

[2] Bender, C. and Elliott, R. J. (2003). On the Clark-Ocone theorem for fractional Brownian motions with Hurst parameter bigger than a half. Stoch. Stoch. Reports 75, 391-405.

[3] Biagini, F., Fink, H. ANd KlÜPPElberg, C. (2013). A fractional credit model with long range dependent default rate. Stoch. Process. Appl. 123, 1319-1347.

[4] Buchmann, B. AND KlÜPPELBERG, C. (2006). Fractional integral equations and state space transforms. Bernoulli 12, 431-456.

[5] Dudley, R. M. (2006). Real Analysis and Probability. Cambridge University Press.

[6] Duffie, D. (2004). Credit Risk Modeling with Affine Processes. Scuola Normale Superiore, Pisa.

[7] Duffie, D., Filipovic, D. and Schachermayer, W. (2003). Affine processes and applications in finance. Ann. Appl. Prob. 13, 984-1053.

[8] Duncan, T. E. (2006). Prediction for some processes related to a fractional Brownian motion. Statist. Prob. Lett. 76, 128-134.

[9] Duncan, T. E. ANd Fink, H. (2011). Corrigendum to 'Prediction for some processes related to a fractional Brownian motion'. Statist. Prob. Lett. 81, 1336-1337.

[10] FINK, H. AND KlüPPELBERG, C. (2011). Fractional Lévy driven Ornstein-Uhlenbeck processes and stochastic differential equations. Bernoulli 17, 484-506.

[11] Gripenberg, G. and Norros, I. (1996). On the prediction of fractional Brownian motion. J. Appl. Prob. 33, 400-410.

[12] Guasoni, P., Rásonyi, M. and Schachermayer, W. (2008). Consistent price systems and face-lifting pricing under transaction costs. Ann. Appl. Prob. 18, 491-520.

[13] Guasoni, P., RÁsonyi, M. And Schachermayer, W. (2010). The fundamental theorem of asset pricing for continuous processes under small transaction costs. Ann. Finance 6, 157-191.

[14] Heath, D., Jarrow, R. And Morton, A. (1992). Bond pricing and the term structure of interest rates: a new methodology for contingent claims valuation. Econometrica 60, 77-105. 
[15] Henry, M. And Zaffaroni, P. (2003). The long-range dependence paradigm for macroeconomics and finance. In Theory and Applications of Long-Range Dependence, eds P. Doukhan, G. Oppenheim and M. Taqqu. Birkhäuser, Boston, MA, pp. 417-438.

[16] Krvavich, Y. V. AND Mishura, Y. S. (2001). Differentiability of fractional integrals whose kernels contain fractional Brownian motions. Ukrainian Math. J. 53, 35-47.

[17] Оhashi, A. (2009). Fractional term structure models: no-arbitrage and consistency. Ann. Appl. Prob. 19, 1553-1580.

[18] Pipiras, V. And TaqQu, M. S. (2000). Integration questions related to fractional Brownian motion. Prob. Theory Relat. Fields 118, 251-291.

[19] Pipiras, V. and TaqQu, M. S. (2001). Are classes of deterministic integrals for fractional Brownian motion on an interval complete? Bernoulli 7, 873-897.

[20] Samorodnitsky, G. and Taqqu, M. S. (1994). Stable Non-Gaussian Random Processes. Chapman \& Hall, New York.

[21] Young, L. C. (1936). An inequality of the Hölder type, connected with Stieltjes integration. Acta Math. 67, 251-282.

[22] ZäHLE, M. (1998). Integration with respect to fractal functions and stochastic calculus. I. Prob. Theory Relat. Fields 111, 333-374. 